\title{
Asymptotic Stability of Impulsive Cellular Neural Networks with Infinite Delays via Fixed Point Theory
}

\author{
Yutian Zhang and Yuanhong Guan \\ School of Mathematics and Statistics, Nanjing University of Information Science and Technology, Nanjing 210044, China \\ Correspondence should be addressed to Yutian Zhang; ytzhang81@163.com
}

Received 1 November 2012; Accepted 8 February 2013

Academic Editor: Qi Luo

Copyright ( 2013 Y. Zhang and Y. Guan. This is an open access article distributed under the Creative Commons Attribution License, which permits unrestricted use, distribution, and reproduction in any medium, provided the original work is properly cited.

We employ the new method of fixed point theory to study the stability of a class of impulsive cellular neural networks with infinite delays. Some novel and concise sufficient conditions are presented ensuring the existence and uniqueness of solution and the asymptotic stability of trivial equilibrium at the same time. These conditions are easily checked and do not require the boundedness and differentiability of delays.

\section{Introduction}

Cellular neural networks (CNNs), proposed by Chua and Yang in 1988 [1, 2], have become a hot topic for their numerous successful applications in various fields such as optimization, linear and nonlinear programming, associative memory, pattern recognition, and computer vision.

Due to the finite switching speed of neurons and amplifiers in the implementation of neural networks, it turns out that the time delays should not be neglected, and therefore, the model of delayed cellular neural networks (DCNNs) is put forward, which is naturally of better realistic significances. In fact, besides delay effects, stochastic and impulsive as well as diffusing effects are also likely to exist in neural networks. Accordingly many experts are showing a growing interest in the research on the dynamic behaviors of complex CNNs such as impulsive delayed reaction-diffusion CNNs and stochastic delayed reaction-diffusion CNNs, with a result of many achievements [3-9] obtained.

Synthesizing the reported results about complex CNNs, we find that the existing research methods for dealing with stability are mainly based on Lyapunov theory. However, we also notice that there are still lots of difficulties in the applications of corresponding results to specific problems; correspondingly it is necessary to seek some new techniques to overcome those difficulties.
Encouragingly, in recent few years, Burton and other authors have applied the fixed point theory to investigate the stability of deterministic systems and obtained some more applicable results; for example, see the monograph [10] and papers [11-22]. In addition, more recently, there have been a few publications where the fixed point theory is employed to deal with the stability of stochastic (delayed) differential equations; see [23-29]. Particularly, in [24-26], Luo used the fixed point theory to study the exponential stability of mild solutions to stochastic partial differential equations with bounded delays and with infinite delays. In $[27,28]$, Sakthivel used the fixed point theory to investigate the asymptotic stability in $p$ th moment of mild solutions to nonlinear impulsive stochastic partial differential equations with bounded delays and with infinite delays. In [29], Luo used the fixed point theory to study the exponential stability of stochastic Volterra-Levin equations.

Naturally, for complex CNNs which have high application values, we wonder if we can utilize the fixed point theory to investigate their stability, not just the existence and uniqueness of solution. With this motivation, in the present paper, we aim to discuss the stability of impulsive CNNs with infinite delays via the fixed point theory. It is worth noting that our research skill is the contraction mapping theory which is different from the usual method of Lyapunov theory. We employ the fixed point theorem 
to prove the existence and uniqueness of solution and the asymptotic stability of trivial equilibrium all at once. Some new and concise algebraic criteria are provided, and these conditions are easy to verify and, moreover, do not require the boundedness and differentiability of delays.

\section{Preliminaries}

Let $R^{n}$ denote the $n$-dimensional Euclidean space and let $\|\cdot\|$ represent the Euclidean norm. $\mathcal{N} \triangleq\{1,2, \ldots, n\} . R_{+}=[0, \infty)$. $C[X, Y]$ corresponds to the space of continuous mappings from the topological space $X$ to the topological space $Y$.

In this paper, we consider the following impulsive cellular neural network with infinite delays:

$$
\begin{array}{r}
\frac{\mathrm{d} x_{i}(t)}{\mathrm{d} t}=-a_{i} x_{i}(t)+\sum_{j=1}^{n} b_{i j} f_{j}\left(x_{j}(t)\right) \\
+\sum_{j=1}^{n} c_{i j} g_{j}\left(x_{j}\left(t-\tau_{j}(t)\right)\right), \\
t \geq 0, t \neq t_{k}, \\
\Delta x_{i}\left(t_{k}\right)=x_{i}\left(t_{k}+0\right)-x_{i}\left(t_{k}\right) \\
=I_{i k}\left(x_{i}\left(t_{k}\right)\right), \quad k=1,2, \ldots,
\end{array}
$$

where $i \in \mathcal{N}$ and $n$ is the number of neurons in the neural network. $x_{i}(t)$ corresponds to the state of the $i$ th neuron at time t. $f_{j}(\cdot), g_{j}(\cdot) \in C[R, R]$ denote the activation functions, respectively. $\tau_{j}(t) \in C\left[R_{+}, R_{+}\right]$corresponds to the known transmission delay satisfying $\tau_{j}(t) \rightarrow \infty$ and $t-\tau_{j}(t) \rightarrow \infty$ as $t \rightarrow \infty$. Denote $\vartheta=\inf \left\{t-\tau_{j}(t), t \geq 0, j \in \mathscr{N}\right\}$. The constant $b_{i j}$ represents the connection weight of the $j$ th neuron on the $i$ th neuron at time $t$. The constant $c_{i j}$ denotes the connection strength of the $j$ th neuron on the $i$ th neuron at time $t-\tau_{j}(t)$. The constant $a_{i}>0$ represents the rate with which the ith neuron will reset its potential to the resting state when disconnected from the network and external inputs. The fixed impulsive moments $t_{k}(k=1,2, \ldots)$ satisfy $0=t_{0}<$ $t_{1}<t_{2}<\cdots$ and $\lim _{k \rightarrow \infty} t_{k}=\infty . x_{i}\left(t_{k}+0\right)$ and $x_{i}\left(t_{k}-0\right)$ stand for the right-hand and left-hand limits of $x_{i}(t)$ at time $t_{k}$, respectively. $I_{i k}\left(x_{i}\left(t_{k}\right)\right)$ shows the abrupt change of $x_{i}(t)$ at the impulsive moment $t_{k}$ and $I_{i k}(\cdot) \in C[R, R]$.

Throughout this paper, we always assume that $f_{i}(0)=$ $g_{i}(0)=I_{i k}(0)=0$ for $i \in \mathcal{N}$ and $k=1,2, \ldots$. Thereby, problem (1) and (2) admits a trivial equilibrium $\mathbf{x}=0$.

Denote by $\mathbf{x}(t) \triangleq \mathbf{x}(t ; s, \varphi)=\left(x_{1}\left(t ; s, \varphi_{1}\right), \ldots\right.$, $\left.x_{n}\left(t ; s, \varphi_{n}\right)\right)^{T} \in R^{n}$ the solution to (1) and (2) with the initial condition

$$
x_{i}(s)=\varphi_{i}(s), \quad \vartheta \leq s \leq 0, i \in \mathcal{N}
$$

where $\varphi(s)=\left(\varphi_{1}(s), \ldots, \varphi_{n}(s)\right)^{T} \in R^{n}$ and $\varphi_{i}(s) \in C[[\vartheta, 0]$, $R]$. Denote $|\varphi|=\sup _{s \in[\vartheta, 0]}\|\varphi(s)\|$.

The solution $\mathbf{x}(t) \triangleq \mathbf{x}(t ; s, \varphi) \in R^{n}$ of (1)-(3) is, for the time variable $t$, a piecewise continuous vector-valued function with the first kind discontinuity at the points $t_{k}$ $(k=1,2, \ldots)$, where it is left continuous; that is, the following relations are valid:

$$
\begin{aligned}
x_{i}\left(t_{k}-0\right)= & x_{i}\left(t_{k}\right), \\
x_{i}\left(t_{k}+0\right)= & x_{i}\left(t_{k}\right)+I_{i k}\left(x_{i}\left(t_{k}\right)\right), \\
& \quad i \in \mathcal{N}, k=1,2, \ldots .
\end{aligned}
$$

Definition 1. The trivial equilibrium $\mathbf{x}=0$ is said to be stable, if, for any $\varepsilon>0$, there exists $\delta>0$ such that for any initial condition $\varphi(s) \in C\left[[\vartheta, 0], R^{n}\right]$ satisfying $|\varphi|<\delta$ :

$$
\|\mathbf{x}(t ; s, \varphi)\|<\varepsilon, \quad t \geq 0 .
$$

Definition 2. The trivial equilibrium $\mathbf{x}=0$ is said to be asymptotically stable if the trivial equilibrium $\mathbf{x}=0$ is stable, and for any initial condition $\varphi(s) \in C\left[[\vartheta, 0], R^{n}\right]$, $\lim _{t \rightarrow \infty}\|\mathbf{x}(t ; s, \varphi)\|=0$ holds.

The consideration of this paper is based on the following fixed point theorem.

Theorem 3 (see [30]). Let $\Upsilon$ be a contraction operator on a complete metric space $\Theta$, then there exists a unique point $\zeta \in \Theta$ for which $\Upsilon(\zeta)=\zeta$.

\section{Main Results}

In this section, we will consider the existence and uniqueness of solution and the asymptotic stability of trivial equilibrium by means of the contraction mapping principle. Before proceeding, we introduce some assumptions listed as follows.

(A1) There exist nonnegative constants $l_{j}$ such that, for any $\eta, v \in R$

$$
\left|f_{j}(\eta)-f_{j}(v)\right| \leq l_{j}|\eta-v|, \quad j \in \mathcal{N} .
$$

(A2) There exist nonnegative constants $k_{j}$ such that, for any $\eta, v \in R$

$$
\left|g_{j}(\eta)-g_{j}(v)\right| \leq k_{j}|\eta-v|, \quad j \in \mathcal{N}
$$

(A3) There exist nonnegative constants $p_{j k}$ such that, for any $\eta, v \in R$,

$$
\left|I_{j k}(\eta)-I_{j k}(v)\right| \leq p_{j k}|\eta-v|, \quad j \in \mathcal{N}, k=1,2, \ldots
$$

Let $\mathscr{H}=\mathscr{H}_{1} \times \cdots \times \mathscr{H}_{n}$, and let $\mathscr{H}_{i}(i \in \mathcal{N})$ be the space consisting of functions $\phi_{i}(t):[\vartheta, \infty) \rightarrow R$, where $\phi_{i}(t)$ satisfies the following:

(1) $\phi_{i}(t)$ is continuous on $t \neq t_{k}(k=1,2, \ldots)$;

(2) $\lim _{t \rightarrow t_{k}^{-}} \phi_{i}(t)$ and $\lim _{t \rightarrow t_{k}^{+}} \phi_{i}(t)$ exist; furthermore, $\lim _{t \rightarrow t_{k}^{-}} \phi_{i}(t)=\phi_{i}\left(t_{k}\right)$ for $k=1,2, \ldots$;

(3) $\phi_{i}(s)=\varphi_{i}(s)$ on $s \in[\vartheta, 0]$;

(4) $\phi_{i}(t) \rightarrow 0$ as $t \rightarrow \infty$; 
Abstract and Applied Analysis

3

here $t_{k}(k=1,2, \ldots)$ and $\varphi_{i}(s)(s \in[\vartheta, 0])$ are defined as shown in Section 2. Also $\mathscr{H}$ is a complete metric space when it is equipped with the following metric:

$$
d(\overline{\mathbf{q}}(t), \overline{\mathbf{h}}(t))=\sum_{i=1}^{n} \sup _{t \geq 9}\left|q_{i}(t)-h_{i}(t)\right|,
$$

where $\overline{\mathbf{q}}(t)=\left(q_{1}(t), \ldots, q_{n}(t)\right) \in \mathscr{H}$ and $\overline{\mathbf{h}}(t)=\left(h_{1}(t), \ldots\right.$, $\left.h_{n}(t)\right) \in \mathscr{H}$.

In what follows, we will give the main result of this paper.

Theorem 4. Assume that conditions (A1)-(A3) hold. Provided that

(i) there exists a constant $\mu$ such that $\inf _{k=1,2, \ldots}\left\{t_{k}-t_{k-1}\right\} \geq$ $\mu$,

(ii) there exist constants $p_{i}$ such that $p_{i k} \leq p_{i} \mu$ for $i \in \mathcal{N}$ and $k=1,2, \ldots$,

(iii) $\lambda^{*} \triangleq \sum_{i=1}^{n}\left\{\left(1 / a_{i}\right) \max _{j \in \mathcal{N}}\left|b_{i j} l_{j}\right|+\left(1 / a_{i}\right) \max _{j \in \mathcal{N}}\left|c_{i j} k_{j}\right|\right\}+$ $\max _{i \in \mathcal{N}}\left\{p_{i}\left(\mu+\left(1 / a_{i}\right)\right)\right\}<1$,

(iv) $\max _{i \in \mathcal{N}}\left\{\lambda_{i}\right\}<1 / \sqrt{n}$, where $\lambda_{i}=\left(1 / a_{i}\right) \sum_{j=1}^{n}\left|b_{i j} l_{j}\right|+$ $\left(1 / a_{i}\right) \sum_{j=1}^{n}\left|c_{i j} k_{j}\right|+p_{i}\left(\mu+\left(1 / a_{i}\right)\right)$,

then the trivial equilibrium $\mathbf{x}=0$ is asymptotically stable.

Proof. Multiplying both sides of (1) with $e^{a_{i} t}$ gives, for $t>0$ and $t \neq t_{k}$,

$$
\begin{aligned}
\mathrm{d} e^{a_{i} t} x_{i}(t) & =e^{a_{i} t} \mathrm{~d} x_{i}(t)+a_{i} x_{i}(t) e^{a_{i} t} \mathrm{~d} t \\
= & e^{a_{i} t}\left\{\sum_{j=1}^{n} b_{i j} f_{j}\left(x_{j}(t)\right)\right. \\
& \left.+\sum_{j=1}^{n} c_{i j} g_{j}\left(x_{j}\left(t-\tau_{j}(t)\right)\right)\right\} \mathrm{d} t,
\end{aligned}
$$

which yields after integrating from $t_{k-1}+\varepsilon(\varepsilon>0)$ to $t \in$ $\left(t_{k-1}, t_{k}\right)(k=1,2, \ldots)$

$$
\begin{aligned}
& x_{i}(t) e^{a_{i} t}= x_{i}\left(t_{k-1}+\varepsilon\right) e^{a_{i}\left(t_{k-1}+\varepsilon\right)} \\
&+\int_{t_{k-1}+\varepsilon}^{t} e^{a_{i} s}\left\{\sum_{j=1}^{n} b_{i j} f_{j}\left(x_{j}(s)\right)\right. \\
&\left.+\sum_{j=1}^{n} c_{i j} g_{j}\left(x_{j}\left(s-\tau_{j}(s)\right)\right)\right\} \mathrm{d} s .
\end{aligned}
$$

Letting $\varepsilon \rightarrow 0$ in (11), we have

$$
\begin{aligned}
x_{i}(t) e^{a_{i} t}= & x_{i}\left(t_{k-1}+0\right) e^{a_{i} t_{k-1}} \\
& +\int_{t_{k-1}}^{t} e^{a_{i} s} \\
& \times\left\{\sum_{j=1}^{n} b_{i j} f_{j}\left(x_{j}(s)\right)\right. \\
& \left.+\sum_{j=1}^{n} c_{i j} g_{j}\left(x_{j}\left(s-\tau_{j}(s)\right)\right)\right\} \mathrm{d} s,
\end{aligned}
$$

for $t \in\left(t_{k-1}, t_{k}\right)(k=1,2, \ldots)$. Setting $t=t_{k}-\varepsilon(\varepsilon>0)$ in (12), we get

$$
\begin{aligned}
x_{i}\left(t_{k}-\varepsilon\right) e^{a_{i}\left(t_{k}-\varepsilon\right)} & \\
= & x_{i}\left(t_{k-1}+0\right) e^{a_{i} t_{k-1}} \\
& +\int_{t_{k-1}}^{t_{k}-\varepsilon} e^{a_{i} s}\left\{\sum_{j=1}^{n} b_{i j} f_{j}\left(x_{j}(s)\right)\right. \\
& \left.+\sum_{j=1}^{n} c_{i j} g_{j}\left(x_{j}\left(s-\tau_{j}(s)\right)\right)\right\} \mathrm{d} s,
\end{aligned}
$$

which generates by letting $\varepsilon \rightarrow 0$

$$
\begin{aligned}
x_{i}\left(t_{k}-0\right) e^{a_{i} t_{k}}= & x_{i}\left(t_{k-1}+0\right) e^{a_{i} t_{k-1}} \\
& +\int_{t_{k-1}}^{t_{k}} e^{a_{i} s}\left\{\sum_{j=1}^{n} b_{i j} f_{j}\left(x_{j}(s)\right)\right. \\
& \left.+\sum_{j=1}^{n} c_{i j} g_{j}\left(x_{j}\left(s-\tau_{j}(s)\right)\right)\right\} \mathrm{d} s .
\end{aligned}
$$

Noting $x_{i}\left(t_{k}-0\right)=x_{i}\left(t_{k}\right)$, (14) can be rearranged as

$$
\begin{aligned}
& x_{i}\left(t_{k}\right) e^{a_{i} t_{k}}= x_{i}\left(t_{k-1}+0\right) e^{a_{i} t_{k-1}} \\
&+\int_{t_{k-1}}^{t_{k}} e^{a_{i} s}\left\{\sum_{j=1}^{n} b_{i j} f_{j}\left(x_{j}(s)\right)\right. \\
&\left.+\sum_{j=1}^{n} c_{i j} g_{j}\left(x_{j}\left(s-\tau_{j}(s)\right)\right)\right\} \mathrm{d} s .
\end{aligned}
$$


Combining (12) and (15), we reach that

$$
\begin{aligned}
& x_{i}(t) e^{a_{i} t}= x_{i}\left(t_{k-1}+0\right) e^{a_{i} t_{k-1}} \\
&+\int_{t_{k-1}}^{t} e^{a_{i} s}\left\{\sum_{j=1}^{n} b_{i j} f_{j}\left(x_{j}(s)\right)\right. \\
&\left.+\sum_{j=1}^{n} c_{i j} g_{j}\left(x_{j}\left(s-\tau_{j}(s)\right)\right)\right\} \mathrm{d} s
\end{aligned}
$$

is true for $t \in\left(t_{k-1}, t_{k}\right](k=1,2, \ldots)$. Further,

$$
\begin{aligned}
x_{i}(t) e^{a_{i} t}= & x_{i}\left(t_{k-1}\right) e^{a_{i} t_{k-1}} \\
& +\int_{t_{k-1}}^{t} e^{a_{i} s}\left\{\sum_{j=1}^{n} b_{i j} f_{j}\left(x_{j}(s)\right)\right. \\
& \left.+\sum_{j=1}^{n} c_{i j} g_{j}\left(x_{j}\left(s-\tau_{j}(s)\right)\right)\right\} \mathrm{d} s \\
& +I_{i(k-1)}\left(x_{i}\left(t_{k-1}\right)\right) e^{a_{i} t_{k-1}}
\end{aligned}
$$

holds for $t \in\left(t_{k-1}, t_{k}\right](k=1,2, \cdots)$. Hence,

$$
\begin{aligned}
& x_{i}\left(t_{k-1}\right) e^{a_{i} t_{k-1}=} x_{i}\left(t_{k-2}\right) e^{a_{i} t_{k-2}} \\
&+\int_{t_{k-2}}^{t_{k-1}} e^{a_{i} s}\left\{\sum_{j=1}^{n} b_{i j} f_{j}\left(x_{j}(s)\right)\right. \\
&\left.+\sum_{j=1}^{n} c_{i j} g_{j}\left(x_{j}\left(s-\tau_{j}(s)\right)\right)\right\} \mathrm{d} s \\
&+I_{i(k-2)}\left(x_{i}\left(t_{k-2}\right)\right) e^{a_{i} t_{k-2}}, \\
& x_{i}\left(t_{2}\right) e^{a_{i} t_{2}}= x_{i}\left(t_{1}\right) e^{a_{i} t_{1}} \\
&+\int_{t_{1}}^{t_{2}} e^{a_{i} s}\left\{\sum_{j=1}^{n} b_{i j} f_{j}\left(x_{j}(s)\right)\right. \\
&+I_{i 1}\left(x_{i}\left(t_{1}\right)\right) e^{a_{i} t_{1}}, \\
&\left.+\sum_{j=1}^{n} c_{i j} g_{j}\left(x_{j}\left(s-\tau_{j}(s)\right)\right)\right\} \mathrm{d} s
\end{aligned}
$$

$$
\begin{aligned}
x_{i}\left(t_{1}\right) e^{a_{i} t_{1}}= & \varphi_{i}(0) \\
& +\int_{0}^{t_{1}} e^{a_{i} s}\left\{\sum_{j=1}^{n} b_{i j} f_{j}\left(x_{j}(s)\right)\right. \\
& \left.+\sum_{j=1}^{n} c_{i j} g_{j}\left(x_{j}\left(s-\tau_{j}(s)\right)\right)\right\} \mathrm{d} s,
\end{aligned}
$$

which produces, for $t>0$,

$$
\begin{aligned}
x_{i}(t)= & \varphi_{i}(0) e^{-a_{i} t} \\
& +e^{-a_{i} t} \int_{0}^{t} e^{a_{i} s}\left\{\sum_{j=1}^{n} b_{i j} f_{j}\left(x_{j}(s)\right)\right. \\
& \left.+\sum_{j=1}^{n} c_{i j} g_{j}\left(x_{j}\left(s-\tau_{j}(s)\right)\right)\right\} \mathrm{d} s \\
& +e^{-a_{i} t} \sum_{0<t_{k}<t}\left\{I_{i k}\left(x_{i}\left(t_{k}\right)\right) e^{a_{i} t_{k}}\right\} .
\end{aligned}
$$

Note $x_{i}(0)=\varphi_{i}(0)$ in (19). We then define the following operator $\pi$ acting on $\mathscr{H}$, for $\overline{\mathbf{y}}(t)=\left(y_{1}(t), \ldots, y_{n}(t)\right) \in \mathscr{H}$ :

$$
\pi(\overline{\mathbf{y}})(t)=\left(\pi\left(y_{1}\right)(t), \ldots, \pi\left(y_{n}\right)(t)\right)
$$

where $\pi\left(y_{i}\right)(t):[\vartheta, \infty) \rightarrow R(i \in \mathcal{N})$ obeys the rules as follows:

$$
\begin{aligned}
\pi\left(y_{i}\right)(t)= & \varphi_{i}(0) e^{-a_{i} t} \\
& +e^{-a_{i} t} \int_{0}^{t} e^{a_{i} s}\left\{\sum_{j=1}^{n} b_{i j} f_{j}\left(y_{j}(s)\right)\right. \\
& \left.+\sum_{j=1}^{n} c_{i j} g_{j}\left(y_{j}\left(s-\tau_{j}(s)\right)\right)\right\} \mathrm{d} s \\
& +e^{-a_{i} t} \sum_{0<t_{k}<t}\left\{I_{i k}\left(y_{i}\left(t_{k}\right)\right) e^{a_{i} t_{k}}\right\},
\end{aligned}
$$

on $t \geq 0$ and $\pi\left(y_{i}\right)(s)=\varphi_{i}(s)$ on $s \in[\vartheta, 0]$.

The subsequent part is the application of the contraction mapping principle, which can be divided into two steps.

Step 1. We need to prove $\pi(\mathscr{H}) \subset \mathscr{H}$. Choosing $y_{i}(t) \in \mathscr{H}_{i}$ $(i \in \mathcal{N})$, it is necessary to testify $\pi\left(y_{i}\right)(t) \subset \mathscr{H}_{i}$.

First, since $\pi\left(y_{i}\right)(s)=\varphi_{i}(s)$ on $s \in[\vartheta, 0]$ and $\varphi_{i}(s) \epsilon$ $C[[\vartheta, 0], R]$, we know $\pi\left(y_{i}\right)(s)$ is continuous on $s \in[\vartheta, 0]$. For a fixed time $t>0$, it follows from (21) that

$$
\pi\left(y_{i}\right)(t+r)-\pi\left(y_{i}\right)(t)=Q_{1}+Q_{2}+Q_{3}+Q_{4},
$$


where

$$
\begin{aligned}
Q_{1}= & \varphi_{i}(0) e^{-a_{i}(t+r)}-\varphi_{i}(0) \quad e^{-a_{i} t}, \\
Q_{2}= & e^{-a_{i}(t+r)} \int_{0}^{t+r} e^{a_{i} s} \sum_{j=1}^{n} b_{i j} f_{j}\left(y_{j}(s)\right) \mathrm{d} s \\
& -e^{-a_{i} t} \int_{0}^{t} e^{a_{i} s} \sum_{j=1}^{n} b_{i j} f_{j}\left(y_{j}(s)\right) \mathrm{d} s, \\
Q_{3}= & e^{-a_{i}(t+r)} \int_{0}^{t+r} e^{a_{i} s} \sum_{j=1}^{n} c_{i j} g_{j}\left(y_{j}\left(s-\tau_{j}(s)\right)\right) \mathrm{d} s \\
& -e^{-a_{i} t} \int_{0}^{t} e^{a_{i} s} \sum_{j=1}^{n} c_{i j} g_{j}\left(y_{j}\left(s-\tau_{j}(s)\right)\right) \mathrm{d} s, \\
Q_{4}= & e^{-a_{i}(t+r)} \sum_{0<t_{k}<(t+r)}\left\{I_{i k}\left(y_{i}\left(t_{k}\right)\right) e^{a_{i} t_{k}}\right\} \\
& -e^{-a_{i} t} \sum_{0<t_{k}<t}\left\{I_{i k}\left(y_{i}\left(t_{k}\right)\right) e^{a_{i} t_{k}}\right\} .
\end{aligned}
$$

Owing to $y_{i}(t) \in \mathscr{H}_{i}$, we see that $y_{i}(t)$ is continuous on $t \neq t_{k}(k=1,2, \ldots)$; moreover, $\lim _{t \rightarrow t_{k}^{-}} y_{i}(t)$ and $\lim _{t \rightarrow t_{k}^{+}} y_{i}(t)$ exist, and $\lim _{t \rightarrow t_{k}^{-}} y_{i}(t)=y_{i}\left(t_{k}\right)$.

Consequently, when $t \neq t_{k}(k=1,2, \ldots)$ in (22), it is easy to find that $Q_{i} \rightarrow 0$ as $r \rightarrow 0$ for $i=1, \ldots, 4$, and so $\pi\left(y_{i}\right)(t)$ is continuous on the fixed time $t \neq t_{k}(k=1,2, \ldots)$.

On the other hand, as $t=t_{k}(k=1,2, \ldots)$ in (22), it is not difficult to find that $Q_{i} \rightarrow 0$ as $r \rightarrow 0$ for $i=1,2,3$. Furthermore, if letting $r<0$ be small enough, we derive

$$
\begin{aligned}
Q_{4}= & e^{-a_{i}\left(t_{k}+r\right)} \sum_{0<t_{m}<\left(t_{k}+r\right)} I_{i m}\left(y_{i}\left(t_{m}\right)\right) e^{a_{i} t_{m}} \\
& -e^{-a_{i} t_{k}} \sum_{0<t_{m}<t_{k}} I_{i m}\left(y_{i}\left(t_{m}\right)\right) e^{a_{i} t_{m}} \\
= & \left\{e^{-a_{i}\left(t_{k}+r\right)}-e^{-a_{i} t_{k}}\right\} \\
& \times \sum_{0<t_{m}<t_{k}}\left\{I_{i m}\left(y_{i}\left(t_{m}\right)\right) e^{a_{i} t_{m}}\right\},
\end{aligned}
$$

which implies $\lim _{r \rightarrow 0^{-}} Q_{4}=0$ as $t=t_{k}$. While letting $r>$ 0 tend to zero gives

$$
\begin{aligned}
Q_{4}= & e^{-a_{i}\left(t_{k}+r\right)} \sum_{0<t_{m}<\left(t_{k}+r\right)} I_{i m}\left(y_{i}\left(t_{m}\right)\right) e^{a_{i} t_{m}} \\
& -e^{-a_{i} t_{k}} \sum_{0<t_{m}<t_{k}} I_{i m}\left(y_{i}\left(t_{m}\right)\right) e^{a_{i} t_{m}}
\end{aligned}
$$

$$
\begin{aligned}
= & e^{-a_{i}\left(t_{k}+r\right)}\left\{\sum_{0<t_{m}<t_{k}}\left\{I_{i m}\left(y_{i}\left(t_{m}\right)\right) e^{a_{i} t_{m}}\right\}\right. \\
& \left.+I_{i k}\left(y_{i}\left(t_{k}\right)\right) e^{a_{i} t_{k}}\right\} \\
& -e^{-a_{i} t_{k}} \sum_{0<t_{m}<t_{k}}\left\{I_{i m}\left(y_{i}\left(t_{m}\right)\right) e^{a_{i} t_{m}}\right\} \\
= & \left\{e^{-a_{i}\left(t_{k}+r\right)}-e^{-a_{i} t_{k}}\right\} \\
& \times \sum_{0<t_{m}<t_{k}}\left\{I_{i m}\left(y_{i}\left(t_{m}\right)\right) e^{a_{i} t_{m}}\right\} \\
& +e^{-a_{i}\left(t_{k}+r\right)} I_{i k}\left(y_{i}\left(t_{k}\right)\right) e^{a_{i} t_{k}}
\end{aligned}
$$

which yields $\lim _{r \rightarrow 0^{+}} Q_{4}=e^{-a_{i} t_{k}} I_{i k}\left(y_{i}\left(t_{k}\right)\right) e^{a_{i} t_{k}}$ as $t=t_{k}$.

According to the above discussion, we find that $\pi\left(y_{i}\right)(t)$ : $[\vartheta, \infty) \rightarrow R$ is continuous on $t \neq t_{k}(k=1,2, \ldots)$; moreover, $\lim _{t \rightarrow t_{k}^{-}} \pi\left(y_{i}\right)(t)$ and $\lim _{t \rightarrow t_{k}^{+}} \pi\left(y_{i}\right)(t)$ exist; in addition, $\lim _{t \rightarrow t_{k}^{-}} \pi\left(y_{i}\right)(t)=\pi\left(y_{i}\right)\left(t_{k}\right) \neq \lim _{t \rightarrow t_{k}^{+}} \pi\left(y_{i}\right)(t)$.

Next, we will prove $\pi\left(y_{i}\right)(t) \rightarrow 0$ as $t \rightarrow \infty$. For convenience, denote

$$
\pi\left(y_{i}\right)(t)=J_{1}+J_{2}+J_{3}+J_{4}, \quad t>0,
$$

where $J_{1}=\varphi_{i}(0) e^{-a_{i} t}, J_{2}=e^{-a_{i} t} \int_{0}^{t} e^{a_{i} s} \sum_{j=1}^{n} b_{i j} f_{j}\left(y_{j}(s)\right) \mathrm{d} s$, $J_{4}=e^{-a_{i} t} \sum_{0<t_{k}<t}\left\{I_{i k}\left(y_{i}\left(t_{k}\right)\right) e^{a_{i} t_{k}}\right\}$, and $J_{3}=e^{-a_{i} t} \int_{0}^{t} e^{a_{i} s}$ $\sum_{j=1}^{n} c_{i j} g_{j}\left(y_{j}\left(s-\tau_{j}(s)\right)\right) \mathrm{d} s$.

Due to $y_{j}(t) \in \mathscr{H}_{j}(j \in \mathcal{N})$, we know $\lim _{t \rightarrow \infty} y_{j}(t)=0$. Then for any $\varepsilon>0$, there exists a $T_{j}>0$ such that $t \geq T_{j}$ implies $\left|y_{j}(t)\right|<\varepsilon$. Choose $T^{*}=\max _{j \in \mathscr{N}}\left\{T_{j}\right\}$. It is derived from (A1) that, for $t \geq T^{*}$,

$$
\begin{aligned}
J_{2} \leq & e^{-a_{i} t} \int_{0}^{t} e^{a_{i} s} \sum_{j=1}^{n}\left\{\left|b_{i j} l_{j}\right|\left|y_{j}(s)\right|\right\} \mathrm{d} s \\
= & e^{-a_{i} t} \int_{0}^{T^{*}} e^{a_{i} s} \sum_{j=1}^{n}\left\{\left|b_{i j} l_{j}\right|\left|y_{j}(s)\right|\right\} \mathrm{d} s \\
& +e^{-a_{i} t} \int_{T^{*}}^{t} e^{a_{i} s} \sum_{j=1}^{n}\left\{\left|b_{i j} l_{j}\right|\left|y_{j}(s)\right|\right\} \mathrm{d} s \\
\leq & e^{-a_{i} t} \sum_{j=1}^{n}\left\{\left|b_{i j} l_{j}\right| \sup _{s \in\left[0, T^{*}\right]}\left|y_{j}(s)\right|\right\}\left\{\int_{0}^{T^{*}} e^{a_{i} s} \mathrm{~d} s\right\} \\
& +\varepsilon \sum_{j=1}^{n}\left\{\left|b_{i j} l_{j}\right|\right\} e^{-a_{i} t} \int_{T^{*}}^{t} e^{a_{i} s} \mathrm{~d} s
\end{aligned}
$$




$$
\begin{aligned}
& \leq e^{-a_{i} t} \sum_{j=1}^{n}\left\{\left|b_{i j} l_{j}\right| \sup _{s \in\left[0, T^{*}\right]}\left|y_{j}(s)\right|\right\} \\
& \quad \times\left\{\int_{0}^{T^{*}} e^{a_{i} s} \mathrm{~d} s\right\}+\frac{\varepsilon}{a_{i}} \sum_{j=1}^{n}\left\{\left|b_{i j} l_{j}\right|\right\} .
\end{aligned}
$$

Moreover, as $\lim _{t \rightarrow \infty} e^{-a_{i} t}=0$, we can find a $\overline{\bar{T}}>0$ for the given $\varepsilon$ such that $t \geq \overline{\bar{T}}$ implies $e^{-a_{i} t}<\varepsilon$, which leads to

$$
\begin{aligned}
J_{2} \leq \varepsilon & \left\{\sum_{j=1}^{n}\left\{\left|b_{i j} l_{j}\right| \sup _{s \in\left[0, T^{*}\right]}\left|y_{j}(s)\right|\right\}\right. \\
\times & \left.\left\{\int_{0}^{T^{*}} e^{a_{i} s} d s\right\}+\frac{1}{a_{i}} \sum_{j=1}^{n}\left\{\left|b_{i j} l_{j}\right|\right\}\right\}, \\
t & \geq \max \left\{T^{*}, \overline{\bar{T}}\right\} ;
\end{aligned}
$$

namely,

$$
J_{2} \longrightarrow 0 \text { as } t \longrightarrow \infty
$$

On the other hand, since $t-\tau_{j}(t) \rightarrow \infty$ as $t \rightarrow \infty$, we get $\lim _{t \rightarrow \infty} y_{j}\left(t-\tau_{j}(t)\right)=0$. Then for any $\varepsilon>0$, there also exists a $T_{j}^{\prime}>0$ such that $s \geq T_{j}^{\prime}$ implies $\left|y_{j}\left(s-\tau_{j}(s)\right)\right|<\varepsilon$. Select $\bar{T}=\max _{j \in \mathscr{N}}\left\{T_{j}^{\prime}\right\}$. It follows from (A2) that

$$
\begin{aligned}
& J_{3} \leq e^{-a_{i} t} \int_{0}^{t} e^{a_{i} s} \sum_{j=1}^{n}\left\{\left|c_{i j} k_{j}\right|\left|y_{j}\left(s-\tau_{j}(s)\right)\right|\right\} \mathrm{d} s \\
& =e^{-a_{i} t} \int_{0}^{\bar{T}} e^{a_{i} s} \sum_{j=1}^{n}\left\{\left|c_{i j} k_{j}\right|\left|y_{j}\left(s-\tau_{j}(s)\right)\right|\right\} \mathrm{d} s \\
& +e^{-a_{i} t} \int_{\bar{T}}^{t} e^{a_{i} s} \sum_{j=1}^{n}\left\{\left|c_{i j} k_{j}\right|\left|y_{j}\left(s-\tau_{j}(s)\right)\right|\right\} \mathrm{d} s \\
& \leq \sum_{j=1}^{n}\left\{\left|c_{i j} k_{j}\right| \sup _{s \in[\vartheta, \bar{T}]}\left|y_{j}(s)\right|\right\} e^{-a_{i} t} \int_{0}^{\bar{T}} e^{a_{i} s} \mathrm{~d} s \\
& +\varepsilon \sum_{j=1}^{n}\left\{\left|c_{i j} k_{j}\right|\right\} e^{-a_{i} t} \int_{\bar{T}}^{t} e^{a_{i} s} \mathrm{~d} s \\
& \leq e^{-a_{i} t} \sum_{j=1}^{n}\left\{\left|c_{i j} k_{j}\right| \sup _{s \in[\vartheta, \bar{T}]}\left|y_{j}(s)\right|\right\} \int_{0}^{\bar{T}} e^{a_{i} s} \mathrm{~d} s \\
& +\frac{\varepsilon}{a_{i}} \sum_{j=1}^{n}\left\{\left|c_{i j} k_{j}\right|\right\}
\end{aligned}
$$

which results in

$$
J_{3} \longrightarrow 0 \quad \text { as } t \longrightarrow \infty
$$

Furthermore, from (A3), we know that $\left|I_{i k}\left(y_{i}\left(t_{k}\right)\right)\right| \leq$ $p_{i k}\left|y_{i}\left(t_{k}\right)\right|$. So

$$
J_{4} \leq e^{-a_{i} t} \sum_{0<t_{k}<t}\left\{p_{i k}\left|y_{i}\left(t_{k}\right)\right| e^{a_{i} t_{k}}\right\} .
$$

As $y_{i}(t) \in \mathscr{H}_{i}$, we have $\lim _{t \rightarrow \infty} y_{i}(t)=0$. Then for any $\varepsilon>0$, there exists a nonimpulsive point $T_{i}>0$ such that $s \geq T_{i}$ implies $\left|y_{i}(s)\right|<\varepsilon$. It then follows from conditions (i) and (ii) that

$$
\begin{aligned}
& J_{4} \leq e^{-a_{i} t}\left\{\sum_{0<t_{k}<T_{i}}\left\{p_{i k}\left|y_{i}\left(t_{k}\right)\right| e^{a_{i} t_{k}}\right\}\right. \\
& \left.+\sum_{T_{i}<t_{k}<t}\left\{p_{i k}\left|y_{i}\left(t_{k}\right)\right| e^{a_{i} t_{k}}\right\}\right\} \\
& \leq e^{-a_{i} t} \sum_{0<t_{k}<T_{i}}\left\{p_{i k}\left|y_{i}\left(t_{k}\right)\right| e^{a_{i} t_{k}}\right\} \\
& +e^{-a_{i} t} p_{i} \varepsilon \sum_{T_{i}<t_{k}<t}\left\{\mu e^{a_{i} t_{k}}\right\} \\
& \leq e^{-a_{i} t} \sum_{0<t_{k}<T_{i}}\left\{p_{i k}\left|y_{i}\left(t_{k}\right)\right| e^{a_{i} t_{k}}\right\} \\
& +e^{-a_{i} t} p_{i} \varepsilon\left\{\sum_{T_{i}<t_{r}<t_{k}}\left\{e^{a_{i} t_{r}}\left(t_{r+1}-t_{r}\right)\right\}\right. \\
& \left.+\mu e^{a_{i} t_{k}}\right\} \\
& \leq e^{-a_{i} t} \sum_{0<t_{k}<T_{i}}\left\{p_{i k}\left|y_{i}\left(t_{k}\right)\right| e^{a_{i} t_{k}}\right\} \\
& +e^{-a_{i} t} p_{i} \mathcal{E}\left(\int_{T_{i}}^{t} e^{a_{i} s} \mathrm{~d} s+\mu e^{a_{i} t}\right) \\
& \leq e^{-a_{i} t} \sum_{0<t_{k}<T_{i}}\left\{p_{i k}\left|y_{i}\left(t_{k}\right)\right| e^{a_{i} t_{k}}\right\} \\
& +\frac{\varepsilon p_{i}}{a_{i}}+p_{i} \varepsilon \mu
\end{aligned}
$$

which produces

$$
J_{4} \longrightarrow 0 \quad \text { as } t \longrightarrow \infty \text {. }
$$

From (30), (32), and (35), we deduce $\pi\left(y_{i}\right)(t) \rightarrow 0$ as $t \rightarrow \infty$ for $i \in \mathcal{N}$. We therefore conclude that $\pi\left(y_{i}\right)(t) \subset \mathscr{H}_{i}$ $(i \in \mathscr{N})$ which means $\pi(\mathscr{H}) \subset \mathscr{H}$.

Step 2. We need to prove $\pi$ is contractive. For $\overline{\mathbf{y}}=$ $\left(y_{1}(t), \ldots, y_{n}(t)\right) \in \mathscr{H}$ and $\bar{z}=\left(z_{1}(t), \ldots, z_{n}(t)\right) \in \mathscr{H}$, we estimate

$$
\left|\pi\left(y_{i}\right)(t)-\pi\left(z_{i}\right)(t)\right| \leq I_{1}+I_{2}+I_{3},
$$


where $I_{1}=e^{-a_{i} t} \int_{0}^{t} e^{a_{i} s} \sum_{j=1}^{n}\left[\left|b_{i j} \| f_{j}\left(y_{j}(s)\right)-f_{j}\left(z_{j}(s)\right)\right|\right]$ $\mathrm{d} s, I_{3}=e^{-a_{i} t} \sum_{0<t_{k}<t}\left\{e^{a_{i} t_{k}}\left|I_{i k}\left(y_{i}\left(t_{k}\right)\right)-I_{i k}\left(z_{i}\left(t_{k}\right)\right)\right|\right\}$, and $I_{2}=$ $e^{-a_{i} t} \int_{0}^{t} e^{a_{i} s} \sum_{j=1}^{n}\left[\left|c_{i j}\right|\left|g_{j}\left(y_{j}\left(s-\tau_{j}(s)\right)\right)-g_{j}\left(z_{j}\left(s-\tau_{j}(s)\right)\right)\right|\right] \mathrm{d} s$.

Note

$$
\begin{aligned}
& I_{1} \leq e^{-a_{i} t} \int_{0}^{t} e^{a_{i} s} \sum_{j=1}^{n}\left[\left|b_{i j} l_{j}\right|\left|y_{j}(s)-z_{j}(s)\right|\right] \mathrm{d} s \\
& \leq \max _{j \in \mathcal{N}}\left|b_{i j} l_{j}\right| \sum_{j=1}^{n}\left\{\sup _{s \in[0, t]}\left|y_{j}(s)-z_{j}(s)\right|\right\} e^{-a_{i} t} \int_{0}^{t} e^{a_{i} s} \mathrm{~d} s \\
& \leq \frac{1}{a_{i}} \max _{j \in \mathcal{N}}\left|b_{i j} l_{j}\right| \sum_{j=1}^{n}\left\{\sup _{s \in[0, t]}\left|y_{j}(s)-z_{j}(s)\right|\right\}, \\
& I_{2} \leq e^{-a_{i} t} \int_{0}^{t} e^{a_{i} s} \\
& \times \sum_{j=1}^{n}\left[\left|c_{i j} k_{j}\right| \mid y_{j}\left(s-\tau_{j}(s)\right)\right. \\
& \left.-z_{j}\left(s-\tau_{j}(s)\right) \mid\right] \mathrm{d} s \\
& \leq \max _{j \in \mathcal{N}}\left|c_{i j} k_{j}\right| \sum_{j=1}^{n}\left\{\sup _{s \in[9, t]}\left|y_{j}(s)-z_{j}(s)\right|\right\} e^{-a_{i} t} \int_{0}^{t} e^{a_{i} s} \mathrm{~d} s \\
& \leq \frac{1}{a_{i}} \max _{j \in \mathcal{N}}\left|c_{i j} k_{j}\right| \sum_{j=1}^{n}\left\{\sup _{s \in[9, t]}\left|y_{j}(s)-z_{j}(s)\right|\right\}, \\
& I_{3} \leq e^{-a_{i} t} \sum_{0<t_{k}<t}\left\{e^{a_{i} t_{k}} p_{i k}\left|y_{i}\left(t_{k}\right)-z_{i}\left(t_{k}\right)\right|\right\} \\
& \leq p_{i} e^{-a_{i} t} \sup _{s \in[0, t]}\left|y_{i}(s)-z_{i}(s)\right| \sum_{0<t_{k}<t}\left\{e^{a_{i} t_{k}} \mu\right\} \\
& \leq p_{i} e^{-a_{i} t} \sup _{s \in[0, t]}\left|y_{i}(s)-z_{i}(s)\right| \\
& \times\left\{\sum_{0<t_{r}<t_{k}}\left\{e^{a_{i} t_{r}}\left(t_{r+1}-t_{r}\right)\right\}+e^{a_{i} t_{k}} \mu\right\} \\
& \leq p_{i} \sup _{s \in[0, t]}\left|y_{i}(s)-z_{i}(s)\right| e^{-a_{i} t} \\
& \times\left\{\int_{0}^{t} e^{a_{i} s} \mathrm{~d} s+e^{a_{i} t} \mu\right\} \\
& \leq p_{i}\left(\mu+\frac{1}{a_{i}}\right) \sup _{s \in[0, t]}\left|y_{i}(s)-z_{i}(s)\right| \text {. }
\end{aligned}
$$

It hence follows from (37) that

$$
\begin{gathered}
\left|\pi\left(y_{i}\right)(t)-\pi\left(z_{i}\right)(t)\right| \\
\leq \frac{1}{a_{i}} \max _{j \in \mathcal{N}}\left|b_{i j} l_{j}\right|
\end{gathered}
$$

$$
\begin{aligned}
& \times \sum_{j=1}^{n}\left\{\sup _{s \in[0, t]}\left|y_{j}(s)-z_{j}(s)\right|\right\} \\
& +\frac{1}{a_{i}} \max _{j \in \mathcal{N}}\left|c_{i j} k_{j}\right| \sum_{j=1}^{n}\left\{\sup _{s \in[9, t]}\left|y_{j}(s)-z_{j}(s)\right|\right\} \\
& +p_{i}\left(\mu+\frac{1}{a_{i}}\right) \sup _{s \in[0, t]}\left|y_{i}(s)-z_{i}(s)\right|,
\end{aligned}
$$

which implies

$$
\begin{aligned}
& \sup _{t \in[\vartheta, T]}\left|\pi\left(y_{i}\right)(t)-\pi\left(z_{i}\right)(t)\right| \\
& \leq \frac{1}{a_{i}} \max _{j \in \mathcal{N}}\left|b_{i j} l_{j}\right| \sum_{j=1}^{n}\left\{\sup _{s \in[\vartheta, T]}\left|y_{j}(s)-z_{j}(s)\right|\right\} \\
&+\frac{1}{a_{i}} \max _{j \in \mathcal{N}}\left|c_{i j} k_{j}\right| \sum_{j=1}^{n}\left\{\sup _{s \in[\vartheta, T]}\left|y_{j}(s)-z_{j}(s)\right|\right\} \\
&+p_{i}\left(\mu+\frac{1}{a_{i}}\right) \sup _{s \in[\vartheta, T]}\left|y_{i}(s)-z_{i}(s)\right| .
\end{aligned}
$$

Therefore,

$$
\begin{aligned}
& \sum_{i=1}^{n} \sup _{t \in[-\tau, T]}\left|\pi\left(y_{i}\right)(t)-\pi\left(z_{i}\right)(t)\right| \\
& \quad \leq \lambda^{*} \sum_{j=1}^{n}\left\{\sup _{s \in[\vartheta, T]}\left|y_{j}(s)-z_{j}(s)\right|\right\} .
\end{aligned}
$$

In view of condition (iii), we see $\pi$ is a contraction mapping, and, thus there exists a unique fixed point $\overline{\mathbf{y}}^{*}(\cdot)$ of $\pi$ in $\mathscr{H}$ which means the transposition of $\overline{\mathbf{y}}^{*}(\cdot)$ is the vectorvalued solution to (1)-(3) and its norm tends to zero as $t \rightarrow$ $\infty$.

To obtain the asymptotic stability, we still need to prove that the trivial equilibrium $\mathbf{x}=0$ is stable. For any $\varepsilon>0$, from condition (iv), we can find $\delta$ satisfying $0<\delta<\varepsilon$ such that $\delta+\max _{i \in \mathcal{N}}\left\{\lambda_{i}\right\} \varepsilon \leq \varepsilon / \sqrt{n}$. Let $|\varphi|<\delta$. According to what has been discussed above, we know that there exists a unique solution $\mathbf{x}(t ; s, \varphi)=\left(x_{1}\left(t ; s, \varphi_{1}\right), \ldots, x_{n}\left(t ; s, \varphi_{n}\right)\right)^{T}$ to (1)-(3); moreover,

$$
x_{i}(t)=\pi\left(x_{i}\right)(t)=J_{1}+J_{2}+J_{3}+J_{4}, \quad t \geq 0 ;
$$

here $J_{1}=\varphi_{i}(0) e^{-a_{i} t}, J_{2}=e^{-a_{i} t} \int_{0}^{t} e^{a_{i} s} \sum_{j=1}^{n} b_{i j} f_{j}\left(x_{j}(s)\right) \mathrm{d} s$, $J_{3}=e^{-a_{i} t} \int_{0}^{t} e^{a_{i} s} \sum_{j=1}^{n} c_{i j} g_{j}\left(x_{j}\left(s-\tau_{j}(s)\right)\right) \mathrm{d} s$, and $J_{4}=e^{-a_{i} t}$ $\sum_{0<t_{k}<t}\left\{I_{i k}\left(x_{i}\left(t_{k}\right)\right) e^{a_{i} t_{k}}\right\}$.

Suppose there exists $t^{*}>0$ such that $\left\|\mathbf{x}\left(t^{*} ; s, \varphi\right)\right\|=\varepsilon$ and $\|\mathbf{x}(t ; s, \varphi)\|<\varepsilon$ as $0 \leq t<t^{*}$. It follows from (41) that

$$
\left|x_{i}\left(t^{*}\right)\right| \leq\left|J_{1}\left(t^{*}\right)\right|+\left|J_{2}\left(t^{*}\right)\right|+\left|J_{3}\left(t^{*}\right)\right|+\left|J_{4}\left(t^{*}\right)\right| .
$$


As

$$
\begin{aligned}
\left|J_{1}\left(t^{*}\right)\right| & =\left|\varphi_{i}(0) e^{-a_{i} t^{*}}\right| \leq \delta, \\
\left|J_{2}\left(t^{*}\right)\right| \leq & e^{-a_{i} t^{*}} \int_{0}^{t^{*}} e^{a_{i} s} \sum_{j=1}^{n}\left|b_{i j} l_{j} x_{j}(s)\right| \mathrm{d} s \\
& <\frac{\varepsilon}{a_{i}} \sum_{j=1}^{n}\left|b_{i j} l_{j}\right|, \\
\left|J_{3}\left(t^{*}\right)\right| \leq & e^{-a_{i} t^{*}} \int_{0}^{t^{*}} e^{a_{i} s} \\
& \times \sum_{j=1}^{n}\left|c_{i j} k_{j} x_{j}\left(s-\tau_{j}(s)\right)\right| \mathrm{d} s \\
& <\frac{\varepsilon}{a_{i}} \sum_{j=1}^{n}\left|c_{i j} k_{j}\right|, \\
\leq & \varepsilon p_{i}\left(\mu+\frac{1}{a_{i}}\right), \\
\left|J_{4}\left(t^{*}\right)\right| \leq & p_{i} e^{-a_{i} t^{*}} \sum_{0<t_{k}<t^{*}}\left\{\mu\left|x_{i}\left(t_{k}\right)\right| e^{a_{i} t_{k}}\right\} \\
& \\
& \\
a_{i} t^{*} & \left\{\int_{0}^{t^{*}} e^{a_{i} s} d s+\mu e^{a_{i} t^{*}}\right\}
\end{aligned}
$$

we obtain $\left|x_{i}\left(t^{*}\right)\right|<\delta+\lambda_{i} \varepsilon$.

So $\left\|\mathbf{x}\left(t^{*} ; s, \varphi\right)\right\|^{2}=\sum_{i=1}^{n}\left\{\left|x_{i}\left(t^{*}\right)\right|^{2}\right\}<\sum_{i=1}^{n}\left\{\left|\delta+\lambda_{i} \varepsilon\right|^{2}\right\} \leq$ $n\left|\delta+\max _{i \in \mathcal{N}}\left\{\lambda_{i}\right\} \varepsilon\right|^{2} \leq \varepsilon^{2}$. This contradicts the assumption of $\left\|\mathbf{x}\left(t^{*} ; s, \varphi\right)\right\|=\varepsilon$. Therefore, $\|\mathbf{x}(t ; s, \varphi)\|<\varepsilon$ holds for all $t \geq 0$. This completes the proof.

Corollary 5. Assume that conditions (A1)-(A3) hold. Provided that

(i) $\inf _{k=1,2, \ldots}\left\{t_{k}-t_{k-1}\right\} \geq 1$,

(ii) there exist constants $p_{i}$ such that $p_{i k} \leq p_{i}$ for $i \in \mathcal{N}$ and $k=1,2, \ldots$,

(iii) $\sum_{i=1}^{n}\left\{\left(1 / a_{i}\right) \max _{j \in \mathcal{N}}\left|b_{i j} l_{j}\right|+\left(1 / a_{i}\right) \max _{j \in \mathcal{N}}\left|c_{i j} k_{j}\right|\right\}+$ $\max _{i \in \mathcal{N}}\left\{p_{i}\left(1+\left(1 / a_{i}\right)\right)\right\}<1$,

(iv) $\max _{i \in \mathcal{N}}\left\{\lambda_{i}^{\prime}\right\}<1 / \sqrt{n}$, where $\lambda_{i}^{\prime}=\left(1 / a_{i}\right) \sum_{j=1}^{n}\left|b_{i j} l_{j}\right|+$ $\left(1 / a_{i}\right) \sum_{j=1}^{n}\left|c_{i j} k_{j}\right|+p_{i}\left(1+\left(1 / a_{i}\right)\right)$,

then the trivial equilibrium $\mathbf{x}=0$ is asymptotically stable.

Proof. Corollary 5 is a direct conclusion by letting $\mu=1$ in Theorem 4.

Remark 6. In Theorem 4, we can see it is the fixed point theory that deals with the existence and uniqueness of solution and the asymptotic analysis of trivial equilibrium at the same time, while Lyapunov method fails to do this.

Remark 7. The presented sufficient conditions in Theorems 4 and Corollary 5 do not require even the boundedness and differentiability of delays, let alone the monotone decreasing behavior of delays which is necessary in some relevant works.

Provided that $I_{i k}(\cdot) \equiv 0,(1)$ and (2) will become the following cellular neural network with infinite delays and without impulsive effects:

$$
\begin{array}{r}
\frac{\mathrm{d} x_{i}(t)}{\mathrm{d} t}=-a_{i} x_{i}(t)+\sum_{j=1}^{n} b_{i j} f_{j}\left(x_{j}(t)\right) \\
+\sum_{j=1}^{n} c_{i j} g_{j}\left(x_{j}\left(t-\tau_{j}(t)\right)\right), \\
i \in \mathcal{N}, t \geq 0,
\end{array}
$$

where $a_{i}, b_{i j}, c_{i j}, f_{j}(\cdot), g_{j}(\cdot), \tau_{j}(t)$, and $x_{i}(t)$ are the same as defined in Section 2. Obviously, (44) also admits a trivial equilibrium $\mathbf{x}=0$. From Theorem 4 , we reach the following.

Theorem 8. Assume that conditions (A1)-(A2) hold. Provided that

(i) $\sum_{i=1}^{n}\left\{\left(1 / a_{i}\right) \max _{j \in \mathcal{N}}\left|b_{i j} l_{j}\right|+\left(1 / a_{i}\right) \max _{j \in \mathcal{N}}\left|c_{i j} k_{j}\right|\right\}<1$,

(ii) $\max _{i \in \mathcal{N}}\left\{\lambda_{i}^{\prime \prime}\right\}<1 / \sqrt{n}$, where $\lambda_{i}^{\prime \prime}=\left(1 / a_{i}\right) \sum_{j=1}^{n}\left|b_{i j} l_{j}\right|+$ $\left(1 / a_{i}\right) \sum_{j=1}^{n}\left|c_{i j} k_{j}\right|$,

then the trivial equilibrium $\mathbf{x}=0$ is asymptotically stable.

\section{Example}

Consider the following two-dimensional impulsive cellular neural network with infinite delays:

$$
\begin{aligned}
\frac{\mathrm{d} x_{i}(t)}{\mathrm{d} t}= & -a_{i} x_{i}(t)+\sum_{j=1}^{2} b_{i j} f_{j}\left(x_{j}(t)\right) \\
& +\sum_{j=1}^{2} c_{i j} g_{j}\left(x_{j}\left(t-\tau_{j}(t)\right)\right), \\
& t \geq 0, t \neq t_{k}, \\
\Delta x_{i}\left(t_{k}\right)= & x_{i}\left(t_{k}+0\right)-x_{i}\left(t_{k}\right) \\
= & \arctan \left(0.4 x_{i}\left(t_{k}\right)\right), \quad k=1,2, \ldots,
\end{aligned}
$$

with the initial conditions $x_{1}(s)=\cos (s), x_{2}(s)=\sin (s)$ on $-1 \leq s \leq 0$, where $\tau_{j}(t)=0.4 t+1, a_{1}=a_{2}=7, b_{i j}=0$, $c_{11}=3 / 7, c_{12}=2 / 7, c_{21}=0, c_{22}=1 / 7, f_{j}(s)=g_{j}(s)=$ $(|s+1|-|s-1|) / 2$, and $t_{k}=t_{k-1}+0.5 k$.

It is easy to see that $\mu=0.5, l_{j}=k_{j}=1$, and $p_{i k}=0.4$. Let $p_{i}=0.8$ and compute

$$
\begin{gathered}
\sum_{i=1}^{2}\left\{\frac{1}{a_{i}} \max _{j=1,2}\left|c_{i j} k_{j}\right|\right\}+\max _{i=1,2}\left\{p_{i}\left(\mu+\frac{1}{a_{i}}\right)\right\}<1, \\
\max _{i \in \mathcal{N}}\left\{\lambda_{i}\right\}<\frac{1}{\sqrt{2}},
\end{gathered}
$$


where $\lambda_{i}=\left(1 / a_{i}\right) \sum_{j=1}^{n}\left|c_{i j} k_{j}\right|+p_{i}\left(\mu+\left(1 / a_{i}\right)\right)$. From Theorem 4 , we conclude that the trivial equilibrium $\mathbf{x}=0$ of this twodimensional impulsive cellular neural network with infinite delays is asymptotically stable.

\section{Conclusions}

This work is devoted to seeking new methods to investigate the stability of complex neural networks. From what has been discussed above, we find that the fixed point theory is feasible. With regard to a class of impulsive cellular neural networks with infinite delays, we utilize the contraction mapping principle to deal with the existence and uniqueness of solution and the asymptotic analysis of trivial equilibrium at the same time, for which Lyapunov method feels helpless. Now that there are different kinds of fixed point theorems and complex neural networks, our future work is to continue the study on the application of fixed point theory to the stability analysis of complex neural networks.

\section{Acknowledgment}

This work is supported by the National Natural Science Foundation of China under Grants 60904028, 61174077, and 41105057.

\section{References}

[1] L. O. Chua and L. Yang, "Cellular neural networks: theory," IEEE Transactions on Circuits and Systems, vol. 35, no. 10, pp. 12571272, 1988.

[2] L. O. Chua and L. Yang, "Cellular neural networks: applications," IEEE Transactions on Circuits and Systems, vol. 35, no. 10, pp. 1273-1290, 1988.

[3] G. T. Stamov and I. M. Stamova, "Almost periodic solutions for impulsive neural networks with delay," Applied Mathematical Modelling, vol. 31, no. 7, pp. 1263-1270, 2007.

[4] S. Ahmad and I. M. Stamova, "Global exponential stability for impulsive cellular neural networks with time-varying delays," Nonlinear Analysis: Theory, Methods \& Applications, vol. 69, no. 3, pp. 786-795, 2008.

[5] K. Li, X. Zhang, and Z. Li, "Global exponential stability of impulsive cellular neural networks with time-varying and distributed delay," Chaos, Solitons and Fractals, vol. 41, no. 3, pp. 1427-1434, 2009.

[6] J. Qiu, "Exponential stability of impulsive neural networks with time-varying delays and reaction-diffusion terms," Neurocomputing, vol. 70, no. 4-6, pp. 1102-1108, 2007.

[7] X. Wang and D. Xu, "Global exponential stability of impulsive fuzzy cellular neural networks with mixed delays and reactiondiffusion terms," Chaos, Solitons and Fractals, vol. 42, no. 5, pp. 2713-2721, 2009.

[8] Y. Zhang and Q. Luo, "Global exponential stability of impulsive delayed reaction-diffusion neural networks via Hardy-Poincarè inequality," Neurocomputing, vol. 83, pp. 198-204, 2012.

[9] Y. Zhang and Q. Luo, "Novel stability criteria for impulsive delayed reaction-diffusion Cohen-Grossberg neural networks via Hardy-Poincarè inequality," Chaos, Solitons and Fractals, vol. 45, no. 8, pp. 1033-1040, 2012.
[10] T. A. Burton, Stability by Fixed Point Theory for Functional Differential Equations, Dover, New York, NY, USA, 2006.

[11] L. C. Becker and T. A. Burton, "Stability, fixed points and inverses of delays," Proceedings of the Royal Society of Edinburgh A, vol. 136, no. 2, pp. 245-275, 2006.

[12] T. A. Burton, "Fixed points, stability, and exact linearization," Nonlinear Analysis: Theory, Methods \& Applications, vol. 61, no. 5, pp. 857-870, 2005.

[13] T. A. Burton, "Fixed points, Volterra equations, and Becker's resolvent," Acta Mathematica Hungarica, vol. 108, no. 3, pp. 261281, 2005.

[14] T. A. Burton, "Fixed points and stability of a nonconvolution equation," Proceedings of the American Mathematical Society, vol. 132, no. 12, pp. 3679-3687, 2004.

[15] T. A. Burton, "Perron-type stability theorems for neutral equations," Nonlinear Analysis: Theory, Methods \& Applications, vol. 55, no. 3, pp. 285-297, 2003.

[16] T. A. Burton, "Integral equations, implicit functions, and fixed points," Proceedings of the American Mathematical Society, vol. 124, no. 8, pp. 2383-2390, 1996.

[17] T. A. Burton and T. Furumochi, "Krasnoselskii's fixed point theorem and stability, Nonlinear Analysis: Theory, Methods \& Applications, vol. 49, no. 4, pp. 445-454, 2002.

[18] T. A. Burton and B. Zhang, "Fixed points and stability of an integral equation: nonuniqueness," Applied Mathematics Letters, vol. 17, no. 7, pp. 839-846, 2004.

[19] T. Furumochi, "Stabilities in FDEs by Schauder's theorem," Nonlinear Analysis, Theory, Methods \& Applications, vol. 63, no. 5-7, pp. e217-e224, 2005.

[20] C. Jin and J. Luo, "Fixed points and stability in neutral differential equations with variable delays," Proceedings of the American Mathematical Society, vol. 136, no. 3, pp. 909-918, 2008.

[21] Y. N. Raffoul, "Stability in neutral nonlinear differential equations with functional delays using fixed-point theory," Mathematical and Computer Modelling, vol. 40, no. 7-8, pp. 691-700, 2004.

[22] B. Zhang, "Fixed points and stability in differential equations with variable delays," Nonlinear Analysis: Theory, Methods \& Applications, vol. 63, no. 5-7, pp. e233-e242, 2005.

[23] J. Luo, "Fixed points and stability of neutral stochastic delay differential equations," Journal of Mathematical Analysis \& Applications, vol. 334, no. 1, pp. 431-440, 2007.

[24] J. Luo, "Fixed points and exponential stability of mild solutions of stochastic partial differential equations with delays," Journal of Mathematical Analysis and Applications, vol. 342, no. 2, pp. 753-760, 2008.

[25] J. Luo, "Stability of stochastic partial differential equations with infinite delays," Journal of Computational and Applied Mathematics, vol. 222, no. 2, pp. 364-371, 2008.

[26] J. Luo and T. Taniguchi, "Fixed points and stability of stochastic neutral partial differential equations with infinite delays," Stochastic Analysis and Applications, vol. 27, no. 6, pp. 1163-1173, 2009.

[27] R. Sakthivel and J. Luo, "Asymptotic stability of impulsive stochastic partial differential equations with infinite delays," Journal of Mathematical Analysis and Applications, vol. 356, no. 1, pp. 1-6, 2009.

[28] R. Sakthivel and J. Luo, "Asymptotic stability of nonlinear impulsive stochastic differential equations," Statistics \& Probability Letters, vol. 79, no. 9, pp. 1219-1223, 2009. 
[29] J. Luo, "Fixed points and exponential stability for stochastic Volterra-Levin equations," Journal of Computational and Applied Mathematics, vol. 234, no. 3, pp. 934-940, 2010.

[30] D. R. Smart, Fixed Point Theorems, Cambridge University Press, Cambridge, UK, 1980. 


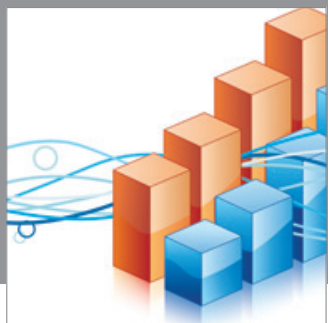

Advances in

Operations Research

mansans

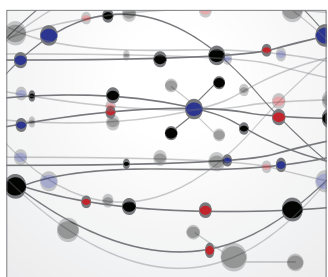

The Scientific World Journal
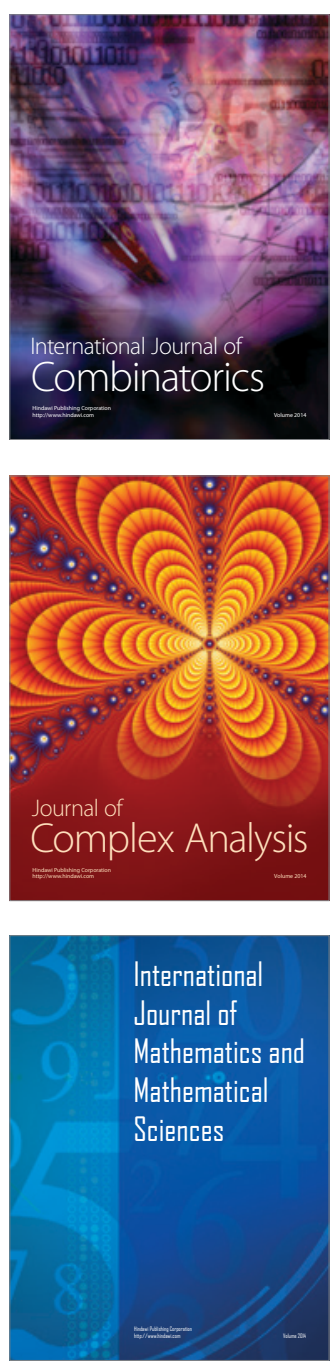
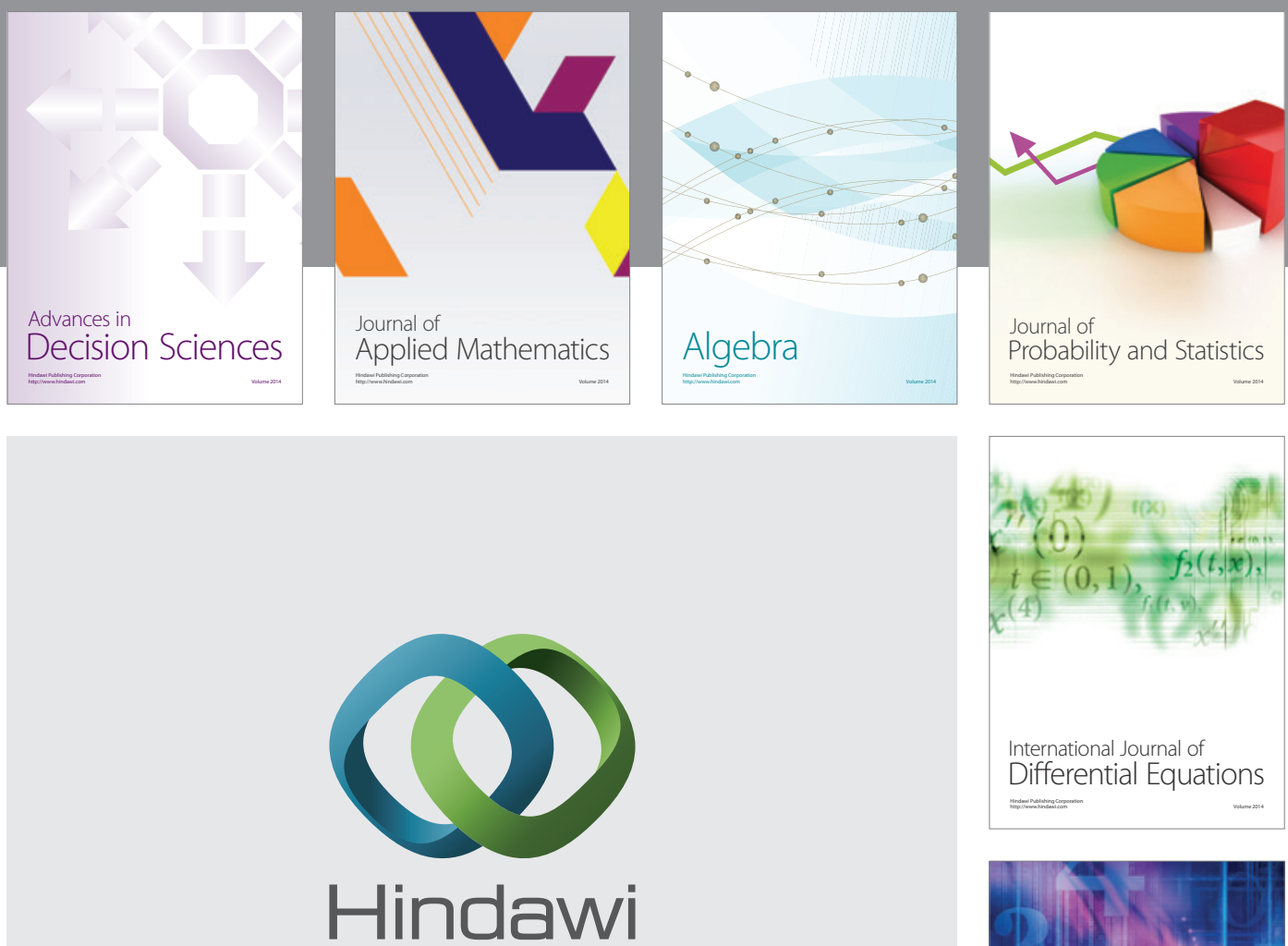

Submit your manuscripts at http://www.hindawi.com
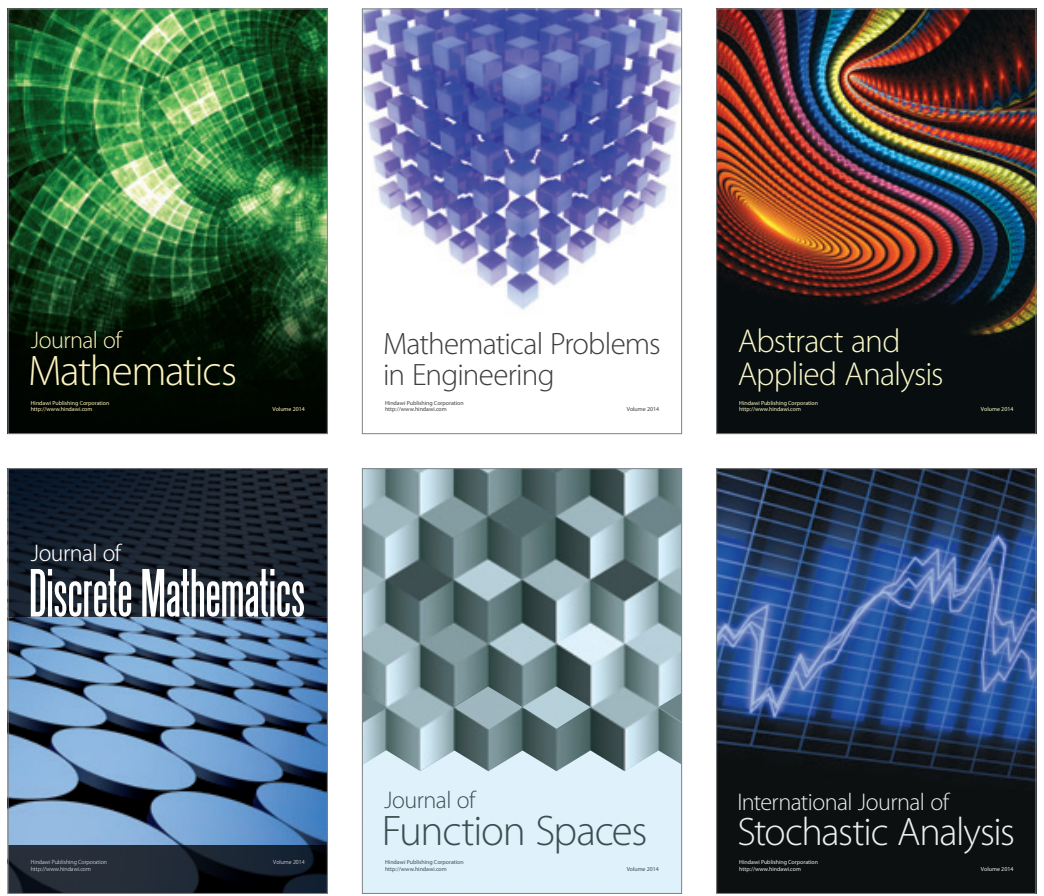

Journal of

Function Spaces

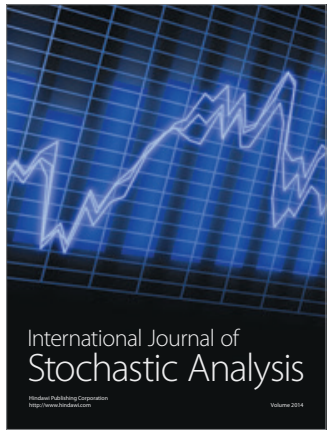

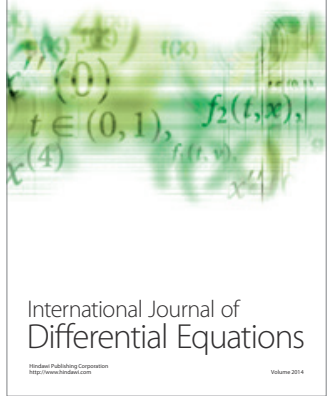
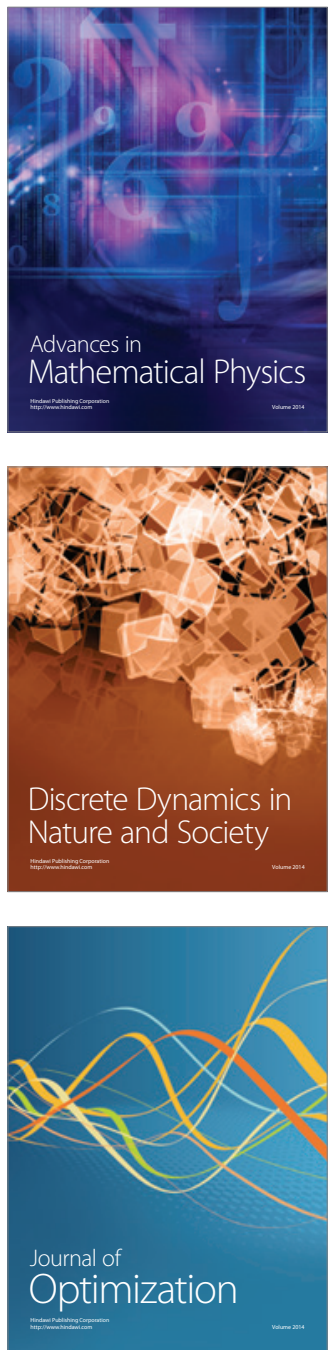